PROCEEDINGS OF THE

AMERICAN MATHEMATICAL SOCIETY

Volume 132, Number 2, Pages 435-441

S 0002-9939(03)07079-5

Article electronically published on June 30, 2003

\title{
MAXIMAL OPERATORS ON SPACES OF HOMOGENEOUS TYPE
}

\author{
GLADIS PRADOLINI AND OSCAR SALINAS
}

(Communicated by Andreas Seeger)

\begin{abstract}
We avoid the assumption given in the work of C. Pérez and R. Wheeden (2001) to prove boundedness properties of certain maximal functions in a general setting of the spaces of homogeneous type with infinite measure. In addition, an example shows that the result can be false if the space has finite measure.
\end{abstract}

\section{INTRODUCTION AND PRELIMINARIES}

In [PW], C. Pérez and R. Wheeden prove some weighted inequalities for potential operators related to a wide class of differential operators called generalized laplacians. This was made in the setting of spaces of homogeneous type. One of the main tools in their proofs is a theorem (Theorem 5.1 in [PW]) about the boundedness properties of a certain maximal function. This theorem was proved by them under the assumption that every annuli is not empty, which implies, for instance, that the space has infinite measure and no atoms (i.e. points with positive measure). In this work we prove that the last assumption can be removed. In particular, we prove that the result is valid in any space of homogeneous type having infinite measure (that is, that the spaces can have atoms). In addition, we give an example showing that the result can be false if the measure of the whole space is finite.

We begin with some definitions.

Let $X$ be a set. A function $d: X \times X \rightarrow \mathbb{R}^{+} \cup\{0\}$ is called a quasi-distance on $X$ if the following conditions are satisfied:

i) for every $x$ and $y$ in $X, d(x, y) \geq 0$, and $d(x, y)=0$ if and only if $x=y$,

ii) for every $x$ and $y$ in $X, d(x, y)=d(y, x)$,

iii) there exists a constant $K$ such that $d(x, y) \leq K(d(x, z)+d(z, y))$ for every $x, y$ and $z$ in $X$.

Let $\mu$ be a positive measure on the $\sigma$-algebra of subsets of $X$ generated by the $d$-balls $B(x, r)=\{y: d(x, y)<r\}$, with $x \in X$ and $r>0$. We assume that $\mu$ satisfies a doubling condition, that is, there exists a constant $A$ such that

$$
0<\mu(B(x, 2 K r)) \leq A \mu(B(x, r))<\infty
$$

Received by the editors July 29, 2002 and, in revised form, September 30, 2002.

2000 Mathematics Subject Classification. Primary 42B25.

Key words and phrases. Maximal operator, spaces of homogeneous type.

The authors were supported by Consejo Nacional de Investigaciones Científicas y Técnicas de la República Argentina and Universidad Nacional del Litoral. 
holds for every ball $B \subset X$. A structure $(X, d, \mu)$, with $d$ and $\mu$ as above, is called a space of homogeneous type. The balls $B(x, r)$ are not necessarily open, but by a result of Macías and Segovia ([MS]), given a quasi-distance $d$ on $X$, it is always possible to find a continuous quasi-distance $d^{\prime}$ on $X$ such that $d^{\prime}$ is equivalent to $d$ (in the sense that there exist two constants $C_{1}$ and $C_{2}$ such that $C_{1} d^{\prime}(x, y) \leq d(x, y) \leq C_{2} d^{\prime}(x, y)$ for all $\left.x, y \in X\right)$. Then, from this and noting that the problem considered here is invariant by changing equivalent quasi-metrics, we can always assume that the quasi-metric $d$ is continuous.

Now, we summarize a few facts about Orlicz spaces. Recall that a function $\phi:[0, \infty) \rightarrow[0, \infty)$ is called a doubling Young function if it is continuous, convex, increasing and satisfying $\phi(0)=0$ and $\phi(t) \rightarrow \infty$ as $t \rightarrow \infty$, and if it satisfies $\phi(2 t) \leq C \phi(t)$. In order to introduce the above-mentioned maximal operator, we set

$$
\|f\|_{\phi, B}=\inf \left\{\lambda>0: \frac{1}{\mu(B)} \int_{B} \phi(|f(y)| / \lambda) d \mu \leq 1\right\} .
$$

With this notation the natural maximal operator associated to the Young function $\phi$ is defined as

$$
M_{\phi} f(x)=\sup _{B: x \in B}\|f\|_{\phi, B},
$$

where the supremum is taken over all balls containing $x$.

Given a Young function $\phi, \tilde{\phi}$ will denote the complementary Young function associated to $\phi$, and it has the property that, for all $t>0$,

$$
t \leq \phi^{-1}(t) \tilde{\phi}^{-1}(t) \leq 2 t .
$$

(1.3) Definition. Let $1<p<\infty$. We say that a doubling Young function $\phi$ satisfies the $B_{p}$ condition if there is a positive constant $c$ such that

$$
\int_{c}^{\infty} \frac{\phi(t)}{t^{p}} \frac{d t}{t} \cong \int_{c}^{\infty}\left(\frac{t^{p^{\prime}}}{\tilde{\phi}(t)}\right)^{p-1} \frac{d t}{t}<\infty .
$$

Now we are able to state our main result.

(1.4) Theorem. Let $1<p<\infty$, let $\phi$ be a doubling Young function and let $(X, d, \mu)$ be a space of homogeneous type with $\mu(X)=\infty$. Then the following statements are equivalent:

i) $\phi \in B_{p}$.

ii) There exists a constant $C$ such that

$$
\int_{X} M_{\phi} f(x)^{p} d \mu(x) \leq C \int_{X}|f(x)|^{p} d \mu(x)
$$

for all nonnegative functions $f$.

iii) There exists a constant $C$ such that

$$
\int_{X} M_{\phi} f(x)^{p} w(x) d \mu(x) \leq C \int_{X}|f(x)|^{p} M w(x) d \mu(x)
$$

for all nonnegative functions $f$ and all weights $w$, where $M$ is the HardyLittlewood maximal operator. 
iv) There exists a constant $C$ such that

$$
\int_{X} M f(x)^{p} \frac{w(x)}{\left(M_{\tilde{\phi}}\left(u^{1 / p}\right)(x)\right)^{p}} d \mu(x) \leq C \int_{X}|f(x)|^{p} \frac{M w(x)}{u(x)} d \mu(x)
$$

for all nonnegative functions $f$ and all weights $w$ and $u$.

(1.6) Remark. As we said in the Introduction, the equivalence between i), ii), iii) and iv) was proved in [PW] (Theorem 1.5) but under stronger conditions on the space. Before that, the theorem was proved in the euclidean context by C. Pérez in [P1], and it was used in order to get sharp two weighted estimates for the HardyLittlewood maximal function. For other applications to different operators from harmonic analysis, see [P2], [P3], [P4], [CP1] and [CP2].

(1.7) Remark. It is important to note that if $\mu(X)<\infty$, then implications i) $\Rightarrow$ ii) $\Rightarrow$ iii) $\Rightarrow$ iv) still hold (the proofs require minor changes). The reciprocal are not true. In fact, in the case $X=\{0,1\}$ with $\mu(\{0\})=\mu(\{1\})=1$ and the euclidean metric, it is very easy to see that $\phi(t)=t^{p}$ verifies ii), but $\phi$ does not satisfy i).

\section{Proofs}

In order to prove Theorem 1.4 we need two technical lemmas. The first one provides us with Calderón-Zygmund decomposition related to Orlicz norms. The second contains the key estimate to avoid the hypothesis of [PW].

(2.1) Lemma. Let $f$ be a nonnegative function belonging to $L^{1}(X)$. Then, given $\sigma>1$, for every $\lambda \geq\|f\|_{\phi, X}$ there exists a sequence $\left\{B_{i}\right\}$ of pairwise disjoint balls such that, if $\tilde{B}_{i}$ is the dilation of $B_{i}$ by $\sigma$, the following statements hold:

$$
\|f\|_{\phi, \tilde{B}_{i}} \leq \lambda<\|f\|_{\phi, B_{i}} .
$$

Proof. The proof follows a similar reasoning, with obvious changes, to the one used by $\mathrm{H}$. Aimar in $\mathrm{A}$ for the case $\phi(t)=t$.

(2.4) Remark. In [PW] a similar result is proved but using the fact that every annulus is not empty.

In order to state the second lemma we define $\delta: X \times X \rightarrow \mathbb{R}^{+} \cup\{0\}$ as

$$
\delta(x, y)= \begin{cases}\mu(B(x, d(x, y))) & \text { if } x \neq y \\ 0 & \text { if } x=y\end{cases}
$$

It is easy to see that the function $\delta$ satisfies

(i) $\delta(x, y) \geq 0$ and $\delta(x, y)=0 \quad$ if and only if $\quad x=y$,

(ii) $\delta(x, y) \leq A \delta(y, x)$ and

(iii) $\delta(x, y) \leq A^{2}(\delta(x, z)+\delta(y, z))$ for every $x, y$, and $z$ in $X$, where $A$ is the constant in (1.1).

We observe that $\delta(x, y)$ does not necessarily satisfy a symmetric condition as $d$. The function $\delta$ is called the nonnecessarily symmetric quasi-distance associated to $(X, d, \mu)$ and was introduced by R. Macías, C. Segovia and J. L. Torrea in MST. We denote by $B_{\delta}(x, r)$ the set $\{y: \delta(x, y)<r\}$. The above conditions on $\delta$ imply the existence of a constant $D$ such that

$$
0<\mu\left(B_{\delta}(x, 2 K r)\right) \leq D \mu\left(B_{\delta}(x, r)\right)<\infty .
$$


In [MST] it is also proved that $\delta$ has the following properties:

(i) $B_{\delta}(x, r)=\{x\}$, if $0<r<\mu(\{x\})$,

(ii) $\mu\left(B_{\delta}(x, r)\right) \leq r$, if $\mu(\{x\}) \leq r$,

(iii) $B_{\delta}(x, r)=X$, if $\mu(X) \leq r$,

(iv) $A^{-2} r \leq \mu\left(B_{\delta}(x, r)\right)$, if $r<\mu(X)$.

Now, related to the $\delta$-balls we get the following lemma (see [BS], too).

(2.7) Lemma. Assuming $\mu(X)=\infty$, there exist two constants $C_{o}$ and $C_{1}$, depending only on the constants of the space $(X, d, \mu)$, such that

$$
\mu\left(B_{\delta}\left(z, C_{o} R\right)-B_{\delta}(z, R)\right) \geq C_{1} R
$$

for every $z$ in $X$ and every $R>\mu(\{z\}) / 2 A^{2}$, where $A$ is the constant in (1.1).

Proof. From the properties of the $\delta$-balls it is not difficult to see that $B_{\delta}\left(z, 4 A^{4} R\right)-$ $B_{\delta}\left(z, 2 A^{2} R\right) \neq \emptyset$ for every $z$ in $X$ and every $R$ verifying $R>\mu(\{z\}) / 2 A^{2}$. So, given $z$ and $R$ in that condition, we can take a point $x_{o}$ in that annulus. Then, considering the $\delta$-ball $B_{o}=B_{\delta}\left(x_{o}, R\right)$, we get

$$
\begin{aligned}
2 A^{2} R & <\delta\left(z, x_{o}\right) \leq A^{2}\left(\delta(z, y)+\delta\left(x_{o}, y\right)\right) \\
& \leq A^{2}(\delta(z, y)+R)
\end{aligned}
$$

for every $y \in B_{o}$. From this, we obtain

$$
\delta(z, y) \geq\left(\frac{2 A^{2}-A^{2}}{A^{2}}\right) R=R
$$

On the other hand

$$
\begin{aligned}
\delta(z, y) & \leq A^{2}\left(\delta\left(z, x_{o}\right)+\delta\left(y, x_{o}\right)\right) \\
& \leq A^{2}\left(4 A^{4} R+A \delta\left(x_{0}, y\right)\right) \\
& \leq A^{2}\left(4 A^{4}+A\right) R,
\end{aligned}
$$

for every $y \in B_{o}$. Then, from these estimates and (2.8), taking $C_{o}=A^{2}\left(4 A^{4}+A\right)$, we have

$$
B_{\delta}\left(x_{o}, R\right) \subset B_{\delta}\left(z, C_{o} R\right)-B_{\delta}(z, R) .
$$

Finally, property (iv) above allows us to obtain

$$
\mu\left(B_{\delta}\left(z, C_{o} R\right)-B_{\delta}(z, R)\right) \geq \frac{R}{A^{2}},
$$

so, taking $C_{1}=1 / A^{2}$, we get our desired result.

Now, we can proceed with the proof of our main result.

Proof of Theorem 1.4. The proofs that i) and iii) imply, respectively, ii) and iv) follow the same reasoning applied in the proof of Theorem (5.1) of [PW]. In addition, assuming ii), the proof that iii) holds can be obtained following again the same reasoning used in [PW] but applying Lemma 2.1] above instead of Lemma 5.2 there. 
Now we are going to prove that iv) implies i). If $w=1$, from the hypotheses we obtain

$$
\int_{X} \frac{M f(x)^{p}}{\left(M_{\tilde{\phi}}\left(u^{1 / p}\right)(x)\right)^{p}} d \mu(x) \leq C \int_{X} \frac{|f(x)|^{p}}{u(x)} d \mu(x) .
$$

Let us take a ball $B_{o}=B(z, R)$ with $z \in X$ and let $f=u=\chi_{B_{o}}$. Then from the above inequality we have

$$
\int_{X} \frac{M f(x)^{p}}{M_{\tilde{\phi}}(f)(x)^{p}} d \mu(x) \leq C .
$$

On the other hand, it is easy to check that

$$
M_{\tilde{\phi}}(f)(x)=\sup _{x \in B} \frac{1}{\tilde{\phi}^{-1}\left(\frac{\mu(B)}{\mu\left(B \cap B_{o}\right)}\right)} .
$$

Let $x \in X$ such that $d(x, z)>\eta R, \eta>1$ large enough. Then it is clear that there exists a positive constant $C$ such that

$$
M_{\tilde{\phi}}(f)(x) \cong \frac{1}{\tilde{\phi}^{-1}(C \mu(B(x, d(x, z))))} .
$$

In a similar way it can be proved that

$$
M\left(\chi_{B_{0}}\right)(x) \cong \frac{1}{\mu(B(x, d(x, z)))} .
$$

Now, we choose $\alpha>1$ such that $\mu(B(z, \alpha \eta R))>\mu(B(z, \eta R))$ (the choice is possible because $\mu(X)=\infty)$. Let $\Omega$ be the set $\Omega=\{x: \mu(B(z, d(z, x))) \geq$ $\mu(B(z, \alpha \eta R))\}$. It is clear that $\Omega \subset\{x / d(z, x)>\eta R\}$. Then, from the above estimates we have

$$
\begin{aligned}
C & \geq \int_{\Omega} \frac{M f(x)^{p}}{M_{\tilde{\phi}} f(x)^{p}} d \mu(x) \\
& \geq \int_{\Omega} \frac{\tilde{\phi}^{-1}(C \mu(B(x, d(x, z))))^{p}}{\mu(B(x, d(x, z)))^{p}} d \mu(x) \\
& \geq \int_{\Omega} \frac{\tilde{\phi}^{-1}(C \mu(B(z, d(x, z))))^{p}}{\mu(B(z, d(x, z)))^{p}} d \mu(x) .
\end{aligned}
$$


Then, taking $R_{o}=\mu(B(z, \alpha \eta R))$, from Lemma 2.7] we get

$$
\begin{aligned}
C & \geq \int_{\Omega} \frac{\tilde{\phi}^{-1}(C \mu(B(z, d(x, z))))^{p}}{\mu(B(z, d(x, z)))^{p}} d \mu(x) \\
& =\sum_{j=0}^{\infty} \int_{C_{o}^{j} R_{o} \leq \mu(B(z, d(x, z)))<C_{o}^{j+1} R_{o}} \frac{\tilde{\phi}^{-1}(C \mu(B(z, d(x, z))))^{p}}{\mu(B(z, d(x, z)))^{p}} d \mu(x) \\
& \geq \sum_{j=0}^{\infty}\left(\frac{\tilde{\phi}^{-1}\left(C C_{o}^{j} R_{o}\right)}{C_{o}^{j+1} R_{o}}\right)^{p} \mu\left(B_{\delta}\left(z, C_{o}^{j+1} R_{o}\right)-B_{\delta}\left(z, C_{o}^{j} R_{o}\right)\right) \\
& \geq C_{1}\left(\frac{C}{C_{o}}\right)^{p} \sum_{j=0}^{\infty} \frac{C_{o}^{j} R_{o}}{\left(\phi^{-1}\left(C C_{o}^{j} R_{o}\right)\right)^{p}} \\
& \geq C_{2} \sum_{j=0}^{\infty} \int_{C C_{o}^{j} R_{o}}^{C C_{o}^{j+1} R_{o}} \frac{t}{\left(\phi^{-1}(t)\right)^{p}} \frac{d t}{t} \\
& =C_{2} \int_{C \mu(B(z, \alpha \eta R))}^{\infty} \frac{t}{\left(\phi^{-1}(t)\right)^{p}} \frac{d t}{t} \\
& \cong \int_{c}^{\infty} \frac{\phi(t)}{t^{p}} \frac{d t}{t},
\end{aligned}
$$

that is, $\phi \in B_{p}$. This completes the proof.

\section{REFERENCES}

[A] Aimar, H.: "Singular integrals and approximate identities on spaces of homogeneous type", Trans. Amer. Math. Soc., Vol. 292, No.1 (1985), pp. 135-153 MR 86m:42022

[BS] Bernardis, A. and Salinas, O.: "Two-weighted inequalities for certain maximal fractional operators on spaces of homogeneous type", Revista de la Unión Matemática Argentina, Vol. 41, 3, (1999) MR 2001e:42024

[CP1] Cruz-Uribe, D. and Pérez, C.: "Two weight extrapolation via the maximal operator", J. Funct. Anal. 174 (2000), no. 1, 1-17. MR 2001g:42040

[CP2] Cruz-Uribe, D. and Pérez, C.: "Sharp two-weight, weak-type norm inequalities for singular integral operators", Math. Res. Lett. 6 (1999), pp. 417-428 MR 2000k:42020

[MS] Macías, R. and Segovia, C.: "Lipschitz functions on spaces of homogeneous type", Advances in Math. 33 (1979) pp. 257-270 MR 81c:32017a

[MST] Macías, R., Segovia, C. and Torrea, J.: "Singular integral operators with non-necessarily bounded kernels on spaces of homogeneous type”, Advances in Math., Vol. 93, No. 1 (1992) MR 93h: 42018

[P1] Pérez, C.: "On sufficient conditions for the boundedness of the Hardy-Littlewood maximal operator between weighted $L^{p}$-spaces with different weights", Proc. London Math. Soc. (3) 71, 1995, pp. 135-157 MR 96k:42023

[P2] Pérez, C.: "Sharp estimates for commutators of singular integrals via iterations of the Hardy-Littlewood maximal function", Journal of Fourier Analysis and Applications, Vol. 3, No. 6, 1997, pp. 743-756 MR 99f:42042

[P3] Pérez, C.: "Sharp weighted inequalities for the vector-valued maximal function", Trans. Amer. Math. Soc., Vol. 352, No. 7 (2000), pp. 3265-3288 MR 2000j:42033

[P4] Pérez, C.: "Two weighted inequalities for potential and fractional type maximal operators", Indiana Univ. Math. J. 43, 1994, pp. 663-683 MR 95m:42028 
[PW] Pérez, C. and Wheeden, R.: "Uncertainty principle estimates for vector fields", Journal of Functional Analysis, 181, 2001, pp. 146-188 MR 2002h:42035

[RR] Rao, M. and Ren, Z.: “Theory of Orlicz spaces", Marcel Dekker, Inc., New York, 1991 MR 92e: 46059

Department of Mathematics, Universidad Nacional del Litoral, Instituto de Matemática Aplicada del Litoral (IMAL), Güemes 3450, 3000 Santa Fe, Argentina

E-mail address: gprado@math.unl.edu.ar

Department of Mathematics, Universidad Nacional del Litoral, Instituto de Matemática Aplicada del Litoral (IMAL), Güemes 3450, 3000 Santa Fe, Argentina

E-mail address: salinas@ceride.gov.ar 